\title{
DIGITAL TERRAIN MODEL RECONSTRUCTION AND PRELIMINARY SCIENTIFIC EXPLORATION PLANNING OF THE CHANG'E 3
}

\author{
J. J. Liu ${ }^{\text {a, }}$, X. Ren ${ }^{\text {a }}$, L. L. Mu ${ }^{\text {a }}$, F. F. Wang ${ }^{\text {b }, ~ W . R . ~ W a n g ~}{ }^{\text {a }}$ X. X. Zhang ${ }^{\text {a }}$, C. L. Li ${ }^{\text {a }}$ \\ ${ }^{a}$ National Astronomical Observatories, Chinese Academy of Sciences - liujj@nao.cas.cn
}

Commission VI, WG VI/4

KEY WORDS: Digital terrain Model, Chang'e-3, Yutu, panoramic camera

\begin{abstract}
:
At 13:11 (GMT) December 14, 2013 Chang'e 3 (CE-3) successfully landed at $19.51^{\circ} \mathrm{W}, 44.12^{\circ} \mathrm{N}$ northwestern Mare Imbrium on the Moon, making it China's first planetary mission to land on a celestial body other than Earth. CE-3 explore comprises a lander and a rover. It carries eight scientific instruments onboard, including the descent camera on the lander, and the panoramic camera on the rover. These cameras imaged the topographic features around the landing site. This paper mainly presents the digital terrain model reconstruction techniques for the panoramic camera. Image pairs obtained during the first lunar day are used to reconstructed 3D Digital Terrain Models of $0.02 \mathrm{~m}$ resolution near observation points E and S3. The maps have been extensively used to support Yutu operations and strategic planning of the mission. The preliminary scientific exploration planning of the Yutu rover for the second lunar day has been made.
\end{abstract}

\section{INTRODUCTION}

At 13:11 (GMT) December 14, 2013 Chang'e 3 (CE-3) successfully landed at $19.51^{\circ} \mathrm{W}, 44.12^{\circ} \mathrm{N}$ northwestern Mare Imbrium on the Moon, making it China's first planetary mission to land on a celestial body other than Earth. Approximately seven hours after landing, the Yutu rover separated from the lander at 20:35 GMT of December 14.

CE-3 explore comprises a lander and a rover. It carries eight scientific instruments onboard, including the descent camera, the terrain camera, the Lunar-based ultraviolet telescope(LUT), and the Extreme ultraviolet(EUV) camera on the lander, and the panoramic camera(PCAM), the lunar ground penetration radar, the alpha particle X-ray spectrometer(APXS), and the infrared spectrometer on the rover. The descent camera, the terrain camera and the panoramic camera imaged the topographic features around the landing site.

Precsie location and topography of the landing site is critical for planetary rover missions. Employing images obtained by the descent camera and stereo images by the panoramic camera, we have located the lander on $1.5 \mathrm{~m} / \mathrm{p}$ Chang'e 2 (CE-2) images and constructed 3D terrain models of the landing area. In this paper, the data processing of the digital terrain model reconstruction and some results are discussed. And also the preliminary scientific exploration planning of the Yutu rover is presented.

\section{DATA PROCESSING AND RESULTS}

The Panoramic cameras are one of the rover's major scientific payloads, equipped with two similar components. The left and right panoramic camera are mounted on both sides of rover mast, along the forward direction. The angles between the camera optical axis are 1 degree, and the relative positions of the two cameras had been accurately calibrated in laboratory.

After the rover mast is fully extended, the camera principal point is about $1.6 \mathrm{~m}$ above the lunar surface. This set of two cameras forms a "binocular" optical instruments. The length between principal points is defined as baseline, similar to the human eye base. Based on the binocular imaging principal, the acquired right and left cameras constitute a stereo image pair. By measuring the homologous points coordinates on the stereo image pair, the parallax are calculated, and the 3D coordinates of target can be reconstructed(Li et al, 2004). The panoramic cameras map the 3D lunar surface around Yutu rover by mast pitching and yawing. Table. 1 shows the main information of the panoramic camera.

\begin{tabular}{|cc|}
\hline Parameter name & Parameter value \\
\hline wavelength & visible \\
imaging mode & color or panchromatic \\
imaging $($ switchable $)$ & $3 \sim \infty$ \\
imaging distance $(\mathrm{m})$ & $2352 \times 1728$ (color) \\
pixel number & $1176 \times 864$ (panchromatic) \\
& 7.4 um $($ color $)$ \\
pixel size & 14.8 um $($ panchromatic $)$ \\
& $19.7^{\circ} \times 14.5^{\circ}$ \\
FOV $\left({ }^{\circ}\right)$ & 10 \\
quantized value $($ bit $)$ &
\end{tabular}

Table 1. Panoramic camera Performance

During Yutu first lunar day observation (December 15-25), the Yutu rover travesed from the lander-rover separation point to make a semi-circle around the lander, allowing the panoramic camera onboard Yutu to obtain color images of the lander. Subsequently the panoramic camera obtained 112 and 56 image pairs of the surrounding terrain at observation points $\mathrm{S}$ and $\mathrm{E} 3$, respectively. Based on these image pairs, we have reconstructed 3D Digital Terrain Models of $0.02 \mathrm{~m}$ resolution near points S(Figure 2) and E3(Figure 3). Data processing in shown in Figure 1. 


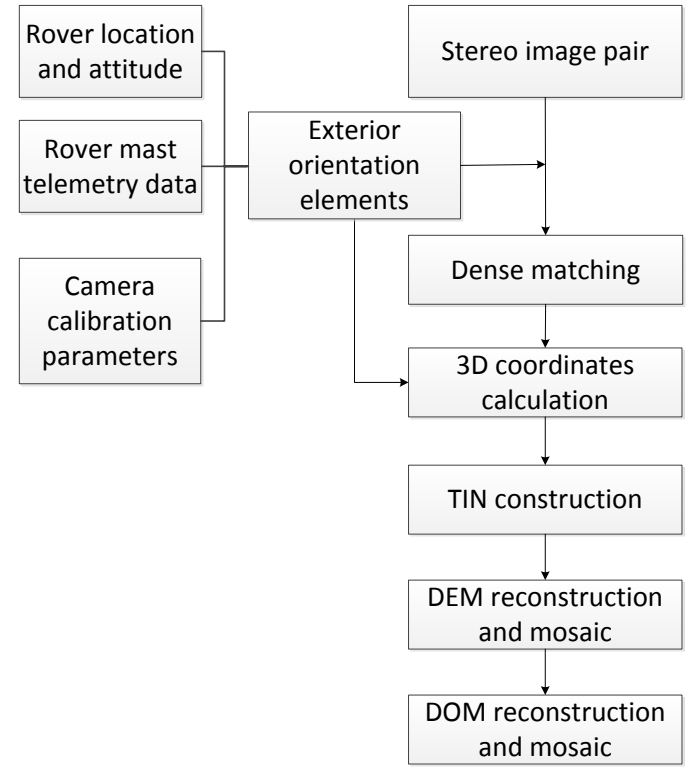

Figure 1. Data Processing Flow Diagram

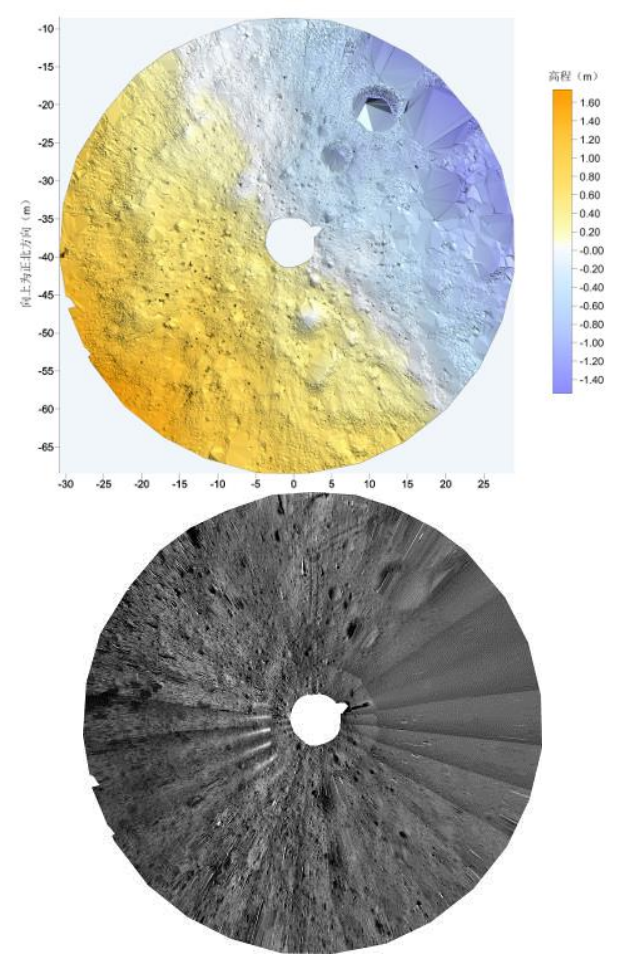

Figure 2. Reconstructed DEM and DOM near points S3
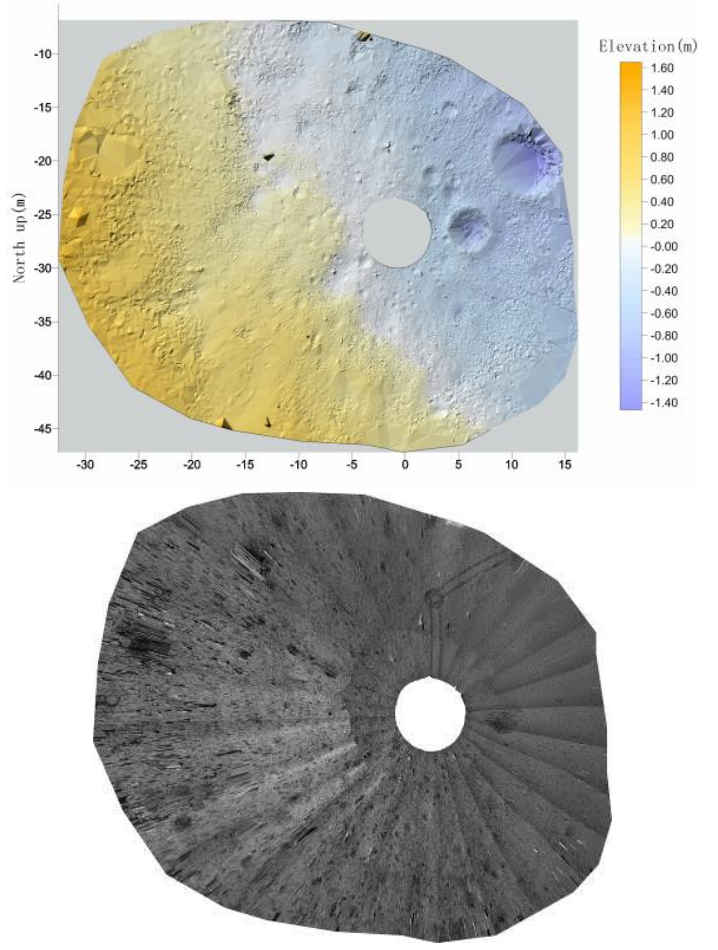

Figure 3. Reconstructed DEM and DOM near points E.

The Rover fell asleep on December 25th, 2013, and completed the exploration mission of the first lunar day. And it is located at $\mathrm{S} 3$ point, about $30 \mathrm{~m}$ south of the lander. With the image data of panoramic camera, terrain camera(TCAM) and landing camera(LCAM) obtained during the first lunar day, we analyzed the terrains, landforms and interesting detection points of the landing site(Tang et al, 2006). Furthermore, we made the scientific plans for the rover detection during the second lunar day(Figure 4).

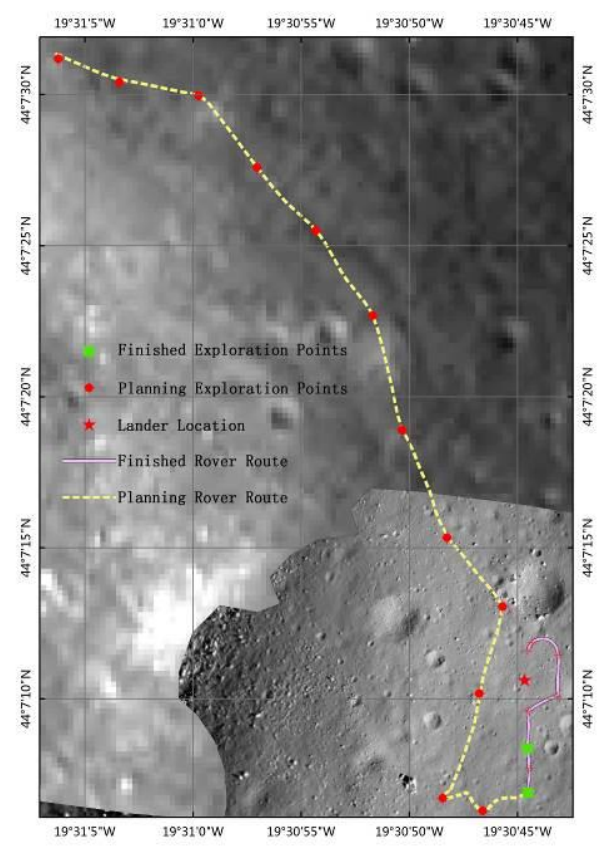

Figure 4. The preliminary scientific exploration planning of the the Yutu rover. 


\section{PRELIMINARY RESULTS}

During its first lunar day observation (December 15-25), the Yutu rover travesed from the lander-rover separation point to make a semi-circle around the lander, allowing the panoramic camera onboard Yutu to obtain color images of the lander. Subsequently the panoramic camera obtained 112 and 56 image pairs of the surrounding terrain at observation points E and S3, respectively. Based on these image pairs, we have reconstructed 3D Digital Terrain Models of $0.02 \mathrm{~m}$ resolution near points $\mathrm{E}$ and S3 (Fig. 2), and made the preliminary scientific exploration planning of the Yutu rover based on these data.

\section{REFERENCES}

K. Di and R. Li, 2004. CAHVOR camera model and its photogrammetric conversion for planetary applications, Journal of Geophysical Research: Planets (1991-2012), vol. 109.

R. Li, K. Di, L. H. Matthies, R. E. Arvidson, W. M. Folkner, and B. A. Archinal, 2004. Rover localization and landing-site mapping technology for the 2003 Mars exploration rover mission, Photogrammetric engineering and remote sensing, vol. 70, pp. 77-90.

Tang Guoan, Liu Xuejun, Lu Guonian, 2006. Digital Elevation Model and Principles and Methodology in Geographical Analysis. Beijing: Science Press.

\section{ACKNOWLEDGEMENTS}

We wish to thank all members corresponding to this study from the Ground Research \&Application System (GRAS), Xi'an Institute of Optics and Precision Mechanics of CAS(XIOPM) and Beijing Geo-Vision Tech.Co.,Ltd. Their joint efforts have made our study possible. 